\title{
HEIDEGGER: ACONTECIMIENTO, DIFERENCIA Y METAFÍSICA
}

\author{
Juan Pablo Esperón* \\ RESUMEN
}

La palabra acontecimiento se muestra como una noción fundamental para la filosofía contemporánea. Pero también resulta ser una noción problemática. El objetivo de este artículo es, entonces, aclarar y mostrar las características que se encuentran sintetizadas en la noción de acontecimiento, con anclaje en la posición teórica de Heidegger, para, en un segundo momento, mostrar algunas dificultades que esta noción conlleva.

Palabras clave: acontecimiento; diferencia; Heidegger; metafísica; problemas

Universidad del Salvador, Universidad Nacional de La Matanza, Conicet, CEF/AncBA, Buenos Aires, Argentina.

Correo electrónico: juanpabloesperon@gmail.com

Para citar este artículo: Esperón, J. P. (2021). Heidegger: acontecimiento, diferencia y metafísica. Universitas Philosophica, 38(76), 79-89. ISSN 0120-5323, ISSN en línea 2346-2426. doi: 10.11144/Javeriana.uph38-76.hadm 


\title{
HEIDEGGER: EVENT, DIFFERENCE AND METAPHYSICS
}

\begin{abstract}
The concept of event is shown as fundamental in contemporary philosophy, but it opens up a problematic field as well. The purpose of this paper is to clarify and discuss the main characteristics of the notion of event, anchored in Heidegger's theoretical stance, and to develop the difficulties that this notion entails.
\end{abstract}

Keywords: event; difference; Heidegger; metaphysics; problems 


\section{Introducción}

Habitamos y vivimos en un mundo cada vez más globalizado, atravesado por el desarrollo tecnológico extremo. Resulta claro que este desarrollo conlleva un gran riesgo para la vida de todos los seres humanos y para el planeta Tierra. En este sentido, resulta imperante para toda la humanidad pensar y construir una nueva vida comunitaria que resguarde y respete también el lugar que nos ha tocado habitar.

Ahora bien, en medio de este contexto signado además por la pandemia de Covid-19, podemos advertir distintas "semillas de futuro" (Scannone, 1967, p. 157) que posibilitan construir una nueva comunidad junto a la Madre Tierra y resignificar el sentido de nuestra existencia individual y colectiva. En este artículo nos proponemos abordar el siguiente problema, eminentemente filosófico, aunque también político: ¿qué es el acontecimiento? ¿Cuáles son sus caracteres fundamentales? ¿Qué conceptos aparecen vinculados a la noción de acontecimiento? ¿Cómo suscitar la ruptura de la causalidad histórica en la que estamos atrapados? ¿Hay alguna relación entre la noción de acontecimiento y el desarrollo tecnocientífico extremo? ¿Es posible construir una nueva relación con la Madre Tierra y la naturaleza no solo a partir de interpretar, sino también de vivenciar, el acontecimiento?

\section{Acontecimiento, diferencia y metafísica}

LA NOCIÓN DE ACONTECIMIENTO tiene un carácter fundamental para la filosofía contemporánea, pero su comprensión acarrea ciertas dificultades. De este modo, resulta importante aclarar su sentido a partir de la posición teórica heideggeriana.

Heidegger postula e introduce, de una manera novedosa para la filosofía, la noción de Ereignis en 1935, cuando escribe los Beiträge zur Philosophie. En esta obra, Heidegger busca encontrar una dimensión originaria y diferente para pensar al ser y su relación con el ente y el Da-sein, alternativa al modo hegemónico de fundamentación metafísica que él denomina "onto-teo-lógica" (Onto-Theologische). Mientras esta última lógica formula y aplica reglas para fundamentar y justificar la totalidad de lo ente en relación con su ser, Heidegger pretende abrir 
un nuevo camino para pensar el ser, el tiempo y su relación con los entes y el hombre. Se trata de postular una nueva manera de pensar la realidad por fuera, o mejor aún, sorteando los límites rígidos establecidos por el paradigma cientificista, por un lado, y los límites del paradigma trascendente-teleológico, por otro.

La noción de acontecimiento en Heidegger está íntimamente vinculada a la noción de diferencia. La diferencia "ontológica" refiere a que el ser es diferente del ente. Pero hay que diferenciar también este sentido del de diferencia "óntica". La diferencia "óntica" refiere a que en la historia de la filosofía -comenzando por Sócrates y Platón, pasando por la filosofía cristiana y la filosofía moderna y culminando con Nietzsche- se confundió al ser con un ente fundamental, con un Ente Supremo (por ejemplo, la Idea, Dios, Razón, Voluntad). Pero Heidegger argumenta que ese ente supremo no es el ser y que su identificación con este se debe a que en la historia de la filosofía hubo una confusión. Al desarrollo histórico de esta confusión, Heidegger lo denominó "historia de la metafísica" y es entendida onto-teo-lógicamente. La metafísica es, entonces, la historia del olvido de la diferencia ontológica a favor de un ente fundamental que justifica y le da entidad a lo ente en general. En la modernidad este ente fundamental es el sujeto, que justifica lo ente, ordenándolo como sistema de causas y efectos. El sujeto se constituye como tal en la medida en que se enfrenta a objetos y los asegura en la representación. Ello resulta posible a partir de la imposición de la física matemática a todo ente cuya base o anclaje es el sujeto moderno. A partir de esto, la naturaleza debe adecuarse a los parámetros impuestos por la física matemática newtoniana. La naturaleza o los entes aparecen como objetos frente al sujeto que, al representárselos, se les ofrece como fundamento. Así, el objeto resulta siempre presentable y completamente a disposición del sujeto. Esta relación de representación constituye un sistema total y cerrado que articula lo real en términos de relaciones de causas y efectos, prefigurando una imagen determinada del mundo. Para Heidegger, esta imagen es la expresión de la voluntad de poder nietzscheana, cuyo poder se efectúa como manipulación y violencia que el sujeto ejerce ante el mundo y sus recursos naturales. Pero, como contracara de esto, paradójicamente, el hombre aparece sujetado por estructuras de dominio institucionales, como tan lúcidamente lo advirtiera Foucault (1989).

Para Heidegger, entonces, este estadio metafísico muestra en el horizonte un límite, puesto que reconocer esta situación actual permite vislumbrar la 
posibilidad de comprender, transitar y también, de alguna manera, asumir esta situación para enfrentarla. Heidegger reconoce y advierte que en el horizonte de comprensión de lo que nombra como Ge-stell ${ }^{1}$ también aparece la dimensión del acontecimiento (Ereignis). Por eso, afirma que

la mutua pertenencia de hombre y ser a modo de provocación alternante, nos muestra sorprendentemente cerca, que de la misma manera que el hombre es dado en propiedad al ser, el ser, por su parte, ha sido atribuido en propiedad al hombre. En la com-posición reina un extraño modo de dar o atribuir propiedad. De lo que se trata es de experimentar sencillamente este juego de apropiación en el que el hombre y el ser se transpropian reciprocamente, esto es, adentrarnos en aquello que nombramos Ereignis (Heidegger, 1976, vol. 11, p. 45; 1990, p. 85).

De este modo, Walton (2018), interpretando las palabras de Heidegger, sostiene que

un cruce entre la yección apropiante del ser y la proyección apropiada del Dasein configura una dimensión por la cual se desoculta el despliegue de la verdad del ser. Así, la yección es delimitada y articulada en una custodia por parte del Dasein, que a la vez se apropia de sí. Pero la proyección puede no apropiarse de la yección, de modo que la verdad del ser se oculta, el Dasein no implementa su pertenencia al ser, y acaece el abandono del ente por el ser. La oscilación entre la yección apropiante del ser y la yección desapropiante, con las que se corresponden la proyección apropiada y la proyección desapropia$\mathrm{da}$, se manifiesta como el conflicto entre el desocultamiento y el ocultamiento de la verdad del ser (pp. 17-18).

Este movimiento que describe Walton configura la estructura tensiva del Ereignis, en cuanto balanceo entre el desocultamiento y el ocultamiento de la verdad del ser; pues la apropiación y expropiación como estructura tensiva del Ereignis da cuenta también de su donación en cuanto despliegue de la verdad del ser.

1 Ge-stell es el nombre con el que Heidegger designa el ser de la técnica en cuanto dominio planetario de todo lo real (no una técnica en particular); sin embargo, este concepto también nombra el límite de la interpretación metafísica del ser como fundamento. 
Por lo anterior, los problemas que Heidegger explicita respecto a la diferencia óntica/ontológica y a la técnica lo conducen a articular un nuevo modo de pensar las diferentes dimensiones de la realidad; es decir, la relación del ser con los entes, el hombre y la naturaleza. Estas relaciones o dimensiones de la realidad serán sintetizadas en la noción de acontecimiento: "el Ereignis es el ámbito en sí mismo oscilante, mediante el cual el hombre y el ser se alcanzan el uno al otro en su esencia y adquieren lo que les es esencial al perder las determinaciones que les prestó la metafísica" (Heidegger, 1976, vol. 11, p. 47; 1990, p. 89). Para Heidegger, entonces, el planteo de la diferencia lo conduce a postular la noción de acontecimiento, porque el ser sobreviene en el ente y lo desoculta, pero, a su vez, el ser se oculta en aquello que desoculta. Esta manifestación del ser, como sobrepasamiento y donación en lo ente al que llega, ad-viene. Aquí aparece una tensión que se "da" entre (Zwischen) ser y ente. Esta tensividad que ocurre en el entre es el acontecimiento. El acontecimiento se dona en este entre como el entrecruzamiento entre la nada, el ser y la temporalidad. Para Heidegger, entonces, el acontecimiento "es la simultaneidad espacio-temporal para el ser (Sein) y el ente" (1976, vol. 65, p. 13; 2003, p. 29). Y el decir acerca de la verdad del ser es "el entre [Das Zwischen] con respecto al esenciarse del ser [Sein] y la entidad del ente. Este "entre" funda la entidad del ente en el ser (Sein)" (1976, vol. 65, p. 13; 2003, p. 29). En suma, para Heidegger, el acontecimiento es la donación de ser y tiempo en su estatus tensivo y diferencial, pues el acontecimiento se dona en la tensión entre el advenimiento del ser y la presencia del ente: diferencia ontológica; pero el mismo acontecimiento no se confunde con la entidad del ente. El Ereignis siempre se retrae y se retira.

3. Recuento de las características sintetizadas en la noción de acontecimiento derivadas de la posición teórica heideggeriana

ANTE TODO, debemos destacar el gran esfuerzo de Heidegger por pensar la realidad de un modo diferente al modelo hegemónico de la filosofía occidental (onto-teo-lógico). A partir de la postulación de las nociones de Ereignis y diferencia, Heidegger brinda elementos para hacer frente al modo metafísico de fundamentación (diferencia óntica); y, por otro lado, abre un nuevo modo de hacer filosofía para el mundo contemporáneo, con anclaje en aquellas nociones. En 
este sentido, Heidegger, desde Sein und Zeit, se da cuenta de que solo se puede decir "hay el ser" (Es gibt Sein) para no caer en la lógica de fundamentación metafísica que combate. Esta expresión apunta al carácter eminentemente donativo del ser, por el cual el ser se dona, abre un claro (Lichtung), irrumpiendo como acontecimiento. Así, el acontecimiento es una forma de autodonación del ser. Esta comprensión del ser como acontecimiento donativo difiere radicalmente del modo en que la tradición metafísica (o como la llama Heidegger, la fundamentación onto-teológica) ha conceptualizado tanto al ser como al tiempo. Por eso, afirma Heidegger que lo que "intentamos pensar es el Es de este Es gibt Sein, Es gibt Zeit, el Es que da ser y tiempo" (1976, vol. 14, p. 23; 2006, p. 24).

A partir de los elementos expuestos, entonces, es posible lograr una caracterización del acontecimiento. En primer lugar, la noción de acontecimiento pareciera ser una noción de muy difícil comprensión, puesto que se halla siempre en tensión: el acontecimiento es irrupción novedosa, pero es también el acontecer temporal en su devenir; emerge a partir de la diferencia y es instancia última de sentido. El acontecimiento no preexiste a ningún "estado de cosas", ni tampoco emerge como una instancia metafísica, trascendente o metahistórica. El acontecimiento solo existe en devenir permanente y abierto y no se identifica con ningún ente.

En segundo lugar, el acontecimiento implica novedad. La irrupción de lo novedoso implica un exceso de sentido, pues da lugar a múltiples y nuevas interpretaciones. La consecuencia directa de esto radica en que la irrupción del acontecimiento resignifica tanto el presente como el pasado y abre inconmensurables posibilidades hacia el futuro. Por consiguiente, el acontecimiento irrumpe como exceso de sentido, y ello implica, a su vez, la instauración de una nueva temporalidad, porque el acontecimiento marca un corte, suspende el flujo "cronológico" del tiempo; el tiempo se interrumpe, se desquicia (Sztajnszrajber, 2020, junio 8).

Por otro lado, el acontecimiento implica singularidad. En el acontecimiento está presente la manifestación entitativa del ente, pero no se identifica con este (diferencia ontológica). Sin embargo, esta manifestación resignifica pasado y futuro. Por lo tanto, una de las características fundamentales del acontecimiento es provocar un cambio radical en el statu quo.

En suma, el acontecimiento se inscribe en una dimensión preontológica, porque el acontecimiento puede ser caracterizado como novedoso, singular y 
contingente, antes de cualquier intento de conceptualización. También el acontecimiento se inscribe en una dimensión genética, porque a partir de la disrupción que produce el acontecimiento en el statu quo es posible interpretar de un nuevo modo la génesis de la realidad. En efecto, el acontecimiento nombra la original e inesperada aparición de la novedad, singularidad y contingencia que irrumpe intempestivamente y pone en suspenso a la sucesión normal de los hechos, es decir, instaura el sentido, o mejor aún, como dice Deleuze (1989), "no hay que preguntar cuál es el sentido de un acontecimiento: el acontecimiento es el mismo sentido" (p. 44). Por esto, Žižek (2014) define al acontecimiento como "algo traumático, perturbador, que parece suceder de repente y que interrumpe el curso normal de las cosas; algo que surge aparentemente de la nada, sin causas discernibles, una apariencia que no tiene como base nada sólido" (p. 16).

De este modo, a partir de los argumentos considerados en este artículo, se puede comprender por qué Scannone sostiene en un artículo titulado El orar como acontecimiento (2017), que el acontecimiento

no solo implica novedad fáctica imprevisible y, por ello, gratuidad, sino que, aunque ilumina el pensamiento, afecta integralmente al ser-ahí y lo funda desde su irreductible diferencia con el mismo evento de donación del ser y con el mismo ser aconteciendo (dándose) como tiempo (p. 19).

\section{Problemas que abre la posición teórica de Heidegger}

LA PRIMERA DIFICULTAD que podemos señalar en el planteo heideggeriano respecto a la cuestión del acontecimiento, es que, este, en cuanto tal, permanece oculto como fenómeno; por lo cual pareciera que habría que estar atentos a sus efectos para desentrañar lo que conlleva, pues como se ha afirmado, el acontecimiento no se confunde con el fenómeno. Por otro lado, si el acontecimiento rompe con todo horizonte de comprensión anterior, ¿es posible interpretarlo desde los marcos teóricos utilizados hasta el momento? ¿No se vuelve toda teoría o presupuesto estéril para comprender al acontecimiento?

Adorno muestra que en la filosofía de Heidegger pareciera haber un equívoco entre el ser y el concepto de ser; pues en el ser se refleja una doble inadecuación. Por un lado, en tanto concepto, es inadecuado para referirse al ser real efectivo, porque el ser nunca se identifica completamente con la cosa a la que 
se refiere. Por otro lado, el ser es un anticoncepto, porque se refiere a algo que no puede ser registrado conceptualmente, es decir, "el concepto de aquello que, por su parte, no puede convertirse en concepto” (Adorno, 2017, p. 125). Por un lado, el ser en cuanto concepto está relegado al lenguaje. Por otro, en tanto es lo que remite a lo que no se agota en el concepto, es lo que no puede ser expresado a través de meros significados. Heidegger no desarrolla estas contradicciones, a saber, "contradicciones como la que hay entre el concepto como simultáneamente anticoncepto o como concepto de lo no conceptual" (Adorno, 2017, p. 116)2.

Por otro lado, desde una perspectiva epistemológica, ¿̇cómo postular una nueva forma de fundamentación para la filosofía y las ciencias si las categorías de las que disponemos para ello son elementos inherentes y constitutivos de esta misma lógica onto-teo-lógica que el discurso de Heidegger pretende superar? Como consecuencia directa de este planteamiento, podemos desprender algunos problemas subsidiarios de esta cuestión: ¿cómo podría ser postulada una ciencia metafísica sin fundamento o, mejor dicho, fundada sobre el acontecimiento y la diferencia?; ¿ cómo integrar la verdad como desocultamiento al programa de esta nueva metafísica? Y también, ¿qué lógicas de investigación se deberían postular para una ciencia fundada en el acontecimiento y la diferencia?

Por último, Deleuze hace una crítica radical respecto de la noción de diferencia en Heidegger. Esta crítica queda formulada explícitamente en Diferencia y repetición (2002), en la nota 21 del primer capítulo, titulado "La diferencia en sí misma”, donde Deleuze sostiene que la metafísica es impotente para pensar la diferencia en cuanto tal, dado que la representación subordina la diferencia a la identidad, pues en la diferencia no hay síntesis, ni mediación, ni reconciliación al modo dialéctico:

la diferencia no implica lo negativo, y no admite ser llevada hasta la contradicción más que en la medida en que se continúe subordinándola a lo idéntico. El primado de la identidad, cualquiera sea la forma en que esta sea concebida, define el mundo de la representación (p. 15).

2 "Heidegger llega al umbral de la comprensión dialéctica de la diferencia dentro de la identidad. Pero no sostiene la contradicción en el concepto de ser. La suprime. [...] La diferencia contenida en la identidad la escamotea como una vergüenza familiar" (Adorno, 1975, p. 107). 
Deleuze tiene presente aquí la posición teórica heideggeriana y discute directamente con él. Para Deleuze, Heidegger sigue pensando al ser y la diferencia con lo ente como arkhé, es decir, con los tintes distintivos de la lógica metafísica tradicional (esto es, como fundamento absoluto, trascendente y teleológico). Entonces, cabe preguntarse ¿Heidegger realmente concibe al ser sustraído de toda subordinación con respecto a la identidad? Deleuze muestra que Heidegger pone en tensión la diferencia con la mismidad (identidad). La diferencia resulta pensada sobre "lo mismo"3 y absorbida por la lógica de la identidad (onto-teológica). Entonces, para Deleuze, Heidegger estaría repitiendo la lógica de la fundamentación metafísica que pretende superar, puesto que en esta filosofía habría una primacía de la identidad sobre la diferencia.

Ahora bien, para Deleuze, replantear de un nuevo modo el problema de la diferencia implica pensar la diferencia en tensión con la repetición, entendida como repetición, no de "lo mismo", sino de la diferencia. Deleuze (1986) reelabora la noción de repetición a partir del concepto de "eterno retorno", que obtiene de su lectura de Nietzsche, de modo tal que, así entendida, se diversifica en multiplicidades siempre originales, y esta potencia activa es lo que se repite eternamente.

Entonces, la postura de Deleuze resulta taxativa respecto a la de Heidegger: postular una filosofía de la diferencia sobre la mismidad impide pensar la diferencia como diferencia. Pues, para Deleuze, pensar la diferencia implica que la realidad tiene una constitución heterogénea; esto significa que la potencia activa del ser distribuye las diferencias, multiplicándolas infinitamente, y aquella potencia retorna eternamente, siendo siempre diferente de sí misma.

Para finalizar, a pesar de las dificultades señaladas, no debemos dejar de reconocer y destacar el esfuerzo que Heidegger hizo para postular un modo alternativo de fundamentación filosófica. No obstante, lo que está en juego en las nociones de acontecimiento y diferencia es la comprensión y el sentido de la realidad que nos toca vivir. La comprensión de una ruptura del curso natural o normal de las cosas, la comprensión de algo inexplicable desde el saber anterior, de un "efecto que parece exceder sus causas", de "un suceso que no está justificado por motivos suficientes" (Žižek, 2014, p. 17). La noción tradicional del "concepto” hace

3 Deleuze discute con Heidegger esta cuestión refiriéndose fundamentalmente a Der Satz der Identität (El principio de identidad; Heidegger, 1976, vol. 11; 1990). 
referencia a la definición, al cierre de la comprensión, pero el acontecimiento señala por el contrario la apertura, lo no clausurable, la irrupción de la novedad. Entonces -se podría objetar-, la novedad, la singularidad y la contingencia del acontecimiento, ¿no harían imposible su aprensión y comprensión? Así es para un autor como Hegel, aun cuando el acontecimiento histórico-universal no escape completamente al concepto por ejemplo, pero el modo de fundamentación filosófica se ha transformado desde entonces y dos siglos después nos encontramos en mejores condiciones para pensar de otro modo la realidad.

Referencias

Adorno, T. (1975). Dialéctica negativa. Madrid: Taurus.

Adorno, T. (2017). Ontología y dialéctica. Lecciones sobre la filosofía de Heidegger. Buenos Aires: Eterna Cadencia.

Deleuze G. (1986). Nietzsche y la filosofía. (Trad. C. Artal). Barcelona: Anagrama.

Deleuze G. (1989). Lógica del sentido. Barcelona: Paidós.

Deleuze G. (2002). Diferencia y repetición. Buenos Aires: Amorrortu.

Foucault, M. (1989). Vigilar y castigar. Nacimiento de la prisión. Buenos Aires: Siglo Veintiuno Editores.

Heidegger, M. (1976). Gesamtausgabe (Vols. 11, 14, 65). Frankfurt am Main: Verlag Vittorio Klostermann.

Heidegger, M. (1990). Identidad y diferencia. Barcelona: Anthropos.

Scannone, J. C. (2017). El orar como acontecimiento. En: J. C. Scannone, R. Walton, J. P. Esperón (Eds.), El acontecimiento y lo sagrado (pp. 17-38). Córdoba: Universidad Católica de Córdoba.

Scannone, J. C. (1967). Situación religiosa actual en América Latina. CIAS. Revista del Centro de Investigación y Acción Social, 55, 149-165.

Sztajnszrajber, D. (2020, junio 8). La pandemia va a terminar, pero el confinamiento va a continuar. Página 12 [revista digital]. Recuperado de: https:// www.pagina12.com.ar/

Walton, R. (2018). Palabras preliminares. En: Basso Monteverde, L. La unidad de la diferencia (pp. 8-25). Buenos Aires: Biblos.

Žižek, S. (2014). Acontecimiento. Madrid: Sexto Piso. 